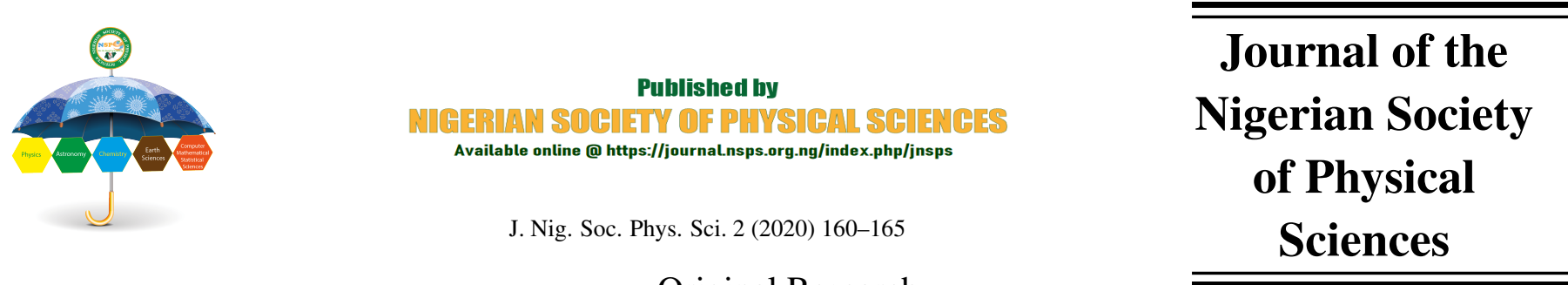

Original Research

\title{
L-Stable Block Hybrid Numerical Algorithm for First-Order Ordinary Differential Equations
}

\author{
B. I. Akinnukawe ${ }^{a, *}$, K. O. Muka ${ }^{b}$ \\ ${ }^{a}$ Department of Mathematics, University of Lagos, Lagos, Nigeria \\ ${ }^{b}$ Department of Mathematics, University of Benin, Benin City, Nigeria
}

\begin{abstract}
In this work, a one-step L-stable Block Hybrid Multistep Method (BHMM) of order five was developed. The method is constructed for solving first order Ordinary Differential Equations with given initial conditions. Interpolation and collocation techniques, with power series as a basis function, are employed for the derivation of the continuous form of the hybrid methods. The discrete scheme and its second derivative are derived by evaluating at the specific grid and off-grid points to form the main and additional methods respectively. Both hybrid methods generated are composed in matrix form and implemented as a block method. The stability and convergence properties of BHMM are discussed and presented. The numerical results of BHMM have proven its efficiency when compared to some existing methods.
\end{abstract}

Keywords: Second derivative, Stability, Hybrid, Block, Collocation techniques

Article History:

Received: 25 May 2020

Received in revised form: 13 July 2020

Accepted for publication: 14 July 2020

Published: 01 August 2020

(c)2020 Journal of the Nigerian Society of Physical Sciences. All rights reserved. Communicated by: J. O. Kuboye

\section{Introduction}

Most Mathematical models are formulated using ordinary differential equations (ODEs) of different orders. However, mathematical models that are based on ODEs of order one occur in several engineering, applied sciences and economics problems. Some of the ODEs have been proven not to have closed-form solution hence the need to develop numerical methods to provide approximate solutions to these problems. Consider the Initial Value Problem of the form

$$
y^{\prime}(x)=f(x, y(x)), \quad y\left(x_{0}\right)=y_{0}
$$

Several numerical methods have been developed by different researchers to circumvent the Dahlquist's order Barrier Theo-

${ }^{*}$ Corresponding author tel. no:

Email address: iakinnukawe@unilag.edu.ng (B. I. Akinnukawe ) rem [1 - 3] that restricted the use of Linear Multistep Methods of high order to solve (1). Some of such researchers are Enright [4], Akinnukawe and Okunuga [5], Gear [6], Okunuga [7], Cash [8], Akinfenwa et al. [9, 10], Adesanya et al. [11], Ijezie and Muka [12] to mention but a few. High-order A-stable and L-stable numerical methods are developed by incorporating off-step points, additional stages and/or employing higher differentiation of the solution (Lambert [13]). Generating approximate solutions for (1) in instances where they exist and are unique but are insoluble via analytical means is very important because they help in the analysis and validation of models in which they evolve from. One of the objectives of developing numerical schemes for solving (1) is to obtain methods with wider stability regions, better convergent rates and computational efficiency. 
In this article, Continuous Hybrid Methods (CHM) of order 5 are derived through Interpolation and Collocation techniques (Onumanyi et al. [14]). Both methods incorporate off-step points and the second derivative of the scheme to produce the discrete hybrid methods which are composed and implemented as a block method (BHMM) to simultaneously produce approximation at nodal points. The BHMM has the advantage of being self-starting, L-stable in nature and possesses high accuracy because it implemented as a block method (see [15], [16], [17]).

\section{Derivation of BHMM}

This section describes the derivation of the k-step hybrid multistep method of the form:

$$
\sum_{j=0}^{k} \alpha_{j} y_{n+j}+\alpha_{v} y_{n+v}=h \sum_{j=0}^{k} \beta_{j} f_{n+j}+h \beta_{v} f_{n+v}+h^{2} \phi_{k} g_{n+k}
$$

where $h, n$ and $v=\frac{1}{2}$ are the step size, grid index and offstep point respectively while $\alpha_{j}, \beta_{j}, j=0,1, \ldots, k$ and $\phi_{k}$ are parameters to be determined uniquely. An approximate solution to (1) by the interpolating function

$$
y(x)=\sum_{j=0}^{4 k+1} b_{j} t^{j}
$$

where $b_{j}, j=0,1, \ldots, 4 k+1$ are the unknown coefficients. Imposing condition for the construction of the proposed class of methods are:

$$
\begin{gathered}
y\left(x_{n+r}\right)=y_{n+r}, \quad r=0, v \\
y^{\prime}\left(x_{n+r}\right)=f_{n+r}, \quad r=0(v) k \\
y^{\prime \prime}\left(x_{n+r}\right)=g_{n+r}, \quad r=k
\end{gathered}
$$

Equations (4) - (6) will lead to a system of $4 k+2$ equations. These equations are solved simultaneously to obtain $b_{j}$ and the values of $b_{j}$ are substituted into (3) to form the Continuous Hybrid Method (CHM) expressed in the form

$$
y(t)=\sum_{j=0}^{k-v} \alpha_{j}(t) y_{n+j}+h \sum_{j=0}^{k} \beta_{j}(t) f_{n+j}+h^{2} \phi_{k}(t) g_{n+k}
$$

where $t=\frac{x-x_{n+k-1}}{h}$ and $\alpha_{j}(t), \beta_{j}(t), j=0(v) k$ and $\phi_{k}(t)$ are the continuous coefficients. The main scheme is generated evaluating CHM (7) at $x_{n+k}$ while the additional scheme is generated from the second derivative of (7) at $x_{n+v}$ of the form

$$
h^{2} y^{\prime \prime}(t)=\sum_{j=0}^{k-v} \overline{\alpha_{j}}(t) y_{n+j}+h \sum_{j=0}^{k} \overline{\beta_{j}}(t) f_{n+j}+h^{2} \overline{\phi_{k}}(t) g_{n+k}
$$

The developed main and additional schemes are combined and implemented simultaneously as a block hybrid multistep method BHMM for the numerical integration of IVPs (1).

Following the steps discussed above, the Continuous Hybrid Method (CHM) for $\mathrm{k}=1$ is equation (7)

$$
y(t)=\sum_{j=0}^{k-v} \alpha_{j}(t) y_{n+j}+h \sum_{j=0}^{k} \beta_{j}(t) f_{n+j}+h^{2} \phi_{k}(t) g_{n+k}
$$

where

$$
\begin{aligned}
& {\left[\begin{array}{c}
\alpha_{0}(t) \\
\alpha_{\frac{1}{2}}(t)
\end{array}\right]=\left[\begin{array}{cccccc}
1 & 0 & -\frac{480}{23} & \frac{1280}{23} & -\frac{1200}{23} & \frac{384}{23} \\
0 & 0 & \frac{480}{23} & -\frac{1280}{23} & \frac{1200}{23} & -\frac{384}{23}
\end{array}\right]\left[\begin{array}{c}
t^{0} \\
t^{1} \\
t^{2} \\
t^{3} \\
t^{4} \\
t^{5}
\end{array}\right](1)} \\
& {\left[\begin{array}{c}
\beta_{0}(t) \\
\beta_{\frac{1}{2}}(t) \\
\beta_{1}(t)
\end{array}\right]=\left[\begin{array}{cccccc}
0 & 1 & -\frac{131}{23} & \frac{265}{23} & -\frac{224}{23} & \frac{68}{23} \\
0 & 0 & -\frac{128}{23} & \frac{464}{23} & -\frac{504}{23} & \frac{176}{23} \\
0 & 0 & \frac{19}{23} & -\frac{89}{23} & \frac{128}{23} & -\frac{52}{23}
\end{array}\right]\left[\begin{array}{c}
t^{0} \\
t^{1} \\
t^{2} \\
t^{3} \\
t^{4} \\
t^{5}
\end{array}\right](2)} \\
& {\left[\begin{array}{lllll}
\phi_{1}(t)
\end{array}\right]=\left[\begin{array}{llllll}
0 & 0 & -\frac{7}{46} & \frac{17}{23} & -\frac{26}{23} & \frac{12}{23}
\end{array}\right]\left[\begin{array}{c}
t^{0} \\
t^{1} \\
t^{2} \\
t^{3} \\
t^{4} \\
t^{5}
\end{array}\right] \quad(3)}
\end{aligned}
$$

Interpolating (7) at $x=x_{n+1}$ to generate the main method at $k=1$ becomes

$y_{n+1}=\frac{7 y_{n}}{23}+\frac{16 y_{n+\frac{1}{2}}}{23}+\frac{h f_{n}}{23}+\frac{8 h f_{n+\frac{1}{2}}}{23}+\frac{6 h f_{n+1}}{23}-\frac{h^{2} g_{n+1}}{46}$

Equation (8), the second derivative of (7) for $k=1$ is

$$
h^{2} y^{\prime \prime}(t)=\sum_{j=0}^{k-v} \overline{\alpha_{j}}(t) y_{n+j}+h \sum_{j=0}^{k} \overline{\beta_{j}}(t) f_{n+j}+h^{2} \overline{\phi_{k}}(t) g_{n+k}
$$

where

$$
\begin{gathered}
{\left[\begin{array}{l}
\overline{\alpha_{0}}(t) \\
\overline{\alpha_{\frac{1}{2}}}(t)
\end{array}\right]=\left[\begin{array}{cccc}
-\frac{960}{23} & \frac{7680}{23} & -\frac{14400}{23} & \frac{7680}{23} \\
\frac{960}{23} & -\frac{7600}{23} & \frac{14400}{23} & -\frac{7680}{23}
\end{array}\right]\left[\begin{array}{c}
t^{0} \\
t^{1} \\
t^{2} \\
t^{3}
\end{array}\right]} \\
{\left[\begin{array}{l}
\overline{\beta_{0}}(t) \\
\overline{\beta_{\frac{1}{2}}}(t) \\
\overline{\beta_{1}}(t)
\end{array}\right]=\left[\begin{array}{cccc}
-\frac{262}{23} & \frac{1590}{23} & -\frac{2688}{23} & \frac{1360}{23} \\
-\frac{256}{23} & \frac{2784}{23} & -\frac{6048}{23} & \frac{3520}{23} \\
\frac{38}{23} & -\frac{534}{23} & \frac{1536}{23} & -\frac{1040}{23}
\end{array}\right]\left[\begin{array}{c}
t^{0} \\
t^{1} \\
t^{2} \\
t^{3}
\end{array}\right]}
\end{gathered}
$$




$$
\left[\overline{\phi_{1}}(t)\right]=\left[\begin{array}{llll}
-\frac{7}{23} & \frac{102}{23} & -\frac{312}{23} & \frac{240}{23}
\end{array}\right]\left[\begin{array}{c}
t^{0} \\
t^{1} \\
t^{2} \\
t^{3}
\end{array}\right]
$$

Interpolating (8) at $x=x_{n+1 / 2}$, the additional method at $k=$ 1 becomes

$h^{2} g_{n+\frac{1}{2}}=\frac{240 y_{n}}{23}-\frac{240 y_{n+\frac{1}{2}}}{23}+\frac{31 h f_{n}}{23}+\frac{64 h f_{n+\frac{1}{2}}}{23}+\frac{25 h f_{n+1}}{23}-\frac{4 h^{2} g_{n+1}}{23}$

The discrete hybrid methods (9a)and (9b) together forms the One-step Block Hybrid Multistep Methods (BHMM). The BHMM can be presented in a matrix block form as

$$
A_{(1)} Y_{\varpi}=A_{(0)} Y_{\varpi-1}+h B_{(1)} F_{\varpi}+h B_{(0)} F_{\varpi-1}+h^{2} C_{(1)} G_{\varpi}
$$

where

$$
\begin{array}{r}
Y_{\varpi}=\left(\begin{array}{c}
y_{n+\frac{1}{2}} \\
y_{n+1}
\end{array}\right) ; Y_{\varpi-1}=\left(\begin{array}{c}
y_{n-\frac{1}{2}} \\
y_{n}
\end{array}\right) ; F_{\varpi}=\left(\begin{array}{c}
f_{n+\frac{1}{2}} \\
f_{n+1}
\end{array}\right) ; \\
F_{\varpi-1}=\left(\begin{array}{c}
f_{n-\frac{1}{2}} \\
f_{n}
\end{array}\right) ; G_{\varpi}=\left(\begin{array}{c}
g_{n+\frac{1}{2}} \\
g_{n+1}
\end{array}\right)
\end{array}
$$

\section{Analysis of BHMM}

\subsection{Order and Error Constant of the Method}

Following Lambert [13] and Fatunla [18], a method was proposed for finding the order $p$ and error constant $W_{p+1}$ of the block method (9) by first expanding $y-, f-$ and $g-$ functions by Taylors series expansion about $x$ and then comparing the coefficients of $h$. It is established from our calculation that one-step Block Hybrid Multistep Method have order and error constants as $p=(5,5)^{T}$ and $W_{p+1}=\left(\frac{13}{44160}, \frac{1}{66240}\right)^{T}$ respectively where $\mathrm{T}$ is transpose.

\subsection{Zero Stability}

A numerical method is said to be zero-stable if the roots $R_{j}, j=1,2, \ldots, N$ of the first characteristic polynomial $\rho(R)$ satisfies $\left|R_{j}\right| \leq 1, j=1, \ldots, N$ and those roots with $\left|R_{j}\right|=1$ is simple (see Lambert [3]). Applying the above conditions to the derived block method, the first characteristic polynomial $\rho(R)=$ 0 is calculated as

$$
\rho(R)=\operatorname{det}\left(R A_{(1)}-A_{(0)}\right)=\frac{240}{23} R(R-1)
$$

The BHMM is found to be zero-stable since $\rho(R)=0$ satisfies $\left|R_{j}\right| \leq 1, j=1,2$.

\subsection{Convergence}

According to Henrici [19], a numerical method converges if it is consistent and zero-stable. Since BHMM (9) is of order

The 2 by 2 matrices $A_{(0)}, A_{(1)}, B_{(0)}, B_{(1)}, C_{(1)}, D_{(1)}$ of the BHMM (9) are defined as follows

$$
\begin{aligned}
& A_{(1)}=\left(\begin{array}{cc}
\frac{240}{23} & 0 \\
-\frac{16}{23} & 1
\end{array}\right) \\
& A_{(0)}=\left(\begin{array}{cc}
0 & \frac{240}{23} \\
0 & \frac{7}{23}
\end{array}\right) \\
& B_{(1)}=\left(\begin{array}{cc}
\frac{64}{23} & \frac{25}{23} \\
\frac{8}{23} & \frac{6}{23}
\end{array}\right) \\
& B_{(0)}=\left(\begin{array}{cc}
0 & \frac{31}{23} \\
0 & \frac{1}{23} \\
0 & -\frac{1}{46} \\
-1 & -\frac{4}{23} \\
0 &
\end{array}\right)
\end{aligned}
$$

$5>1$, then it is consistent and we have established earlier that the method satisfies the conditions of zero-stability. Therefore, the block method (9) converges.

\subsection{Stability of BHMM}

Applying the BHMM to the test equation

$$
y^{\prime}=\lambda y, \quad \lambda \leq 0
$$

we obtain

$$
Y_{\varpi}=Q(z) Y_{\varpi-1}, \quad z=\lambda h
$$

where $Q(z)$ is the amplification matrix given by

$$
Q(z)=\frac{A_{(0)}+z B_{(0)}}{A_{(1)}+z B_{(1)}+z^{2} C_{(1)}}
$$

$Q(z)$ has eigenvalues $\left(\zeta_{1}, \zeta_{2}\right)=\left(0, \zeta_{2}\right)$. The dominant eigenvalue $\zeta_{2}$ is the stability function with real coefficient as

$$
\zeta_{2}=\frac{10.4348+4.17391 z+0.652174 z^{2}+0.0434783 z^{3}}{10.4348-6.26087 z+1.69565 z^{2}-0.26087 z^{3}+0.0217391 z^{4}}
$$

The stability function is used to plot the Region of Absolute Stability (RAS) of the BHMM (see Figure 1). The proposed method BHMM is L-stable since the RAS covers the entire left plane of the graph (A-stable) and the limit of the stability function $\zeta_{2}$ is zero as $z \rightarrow \infty$ 


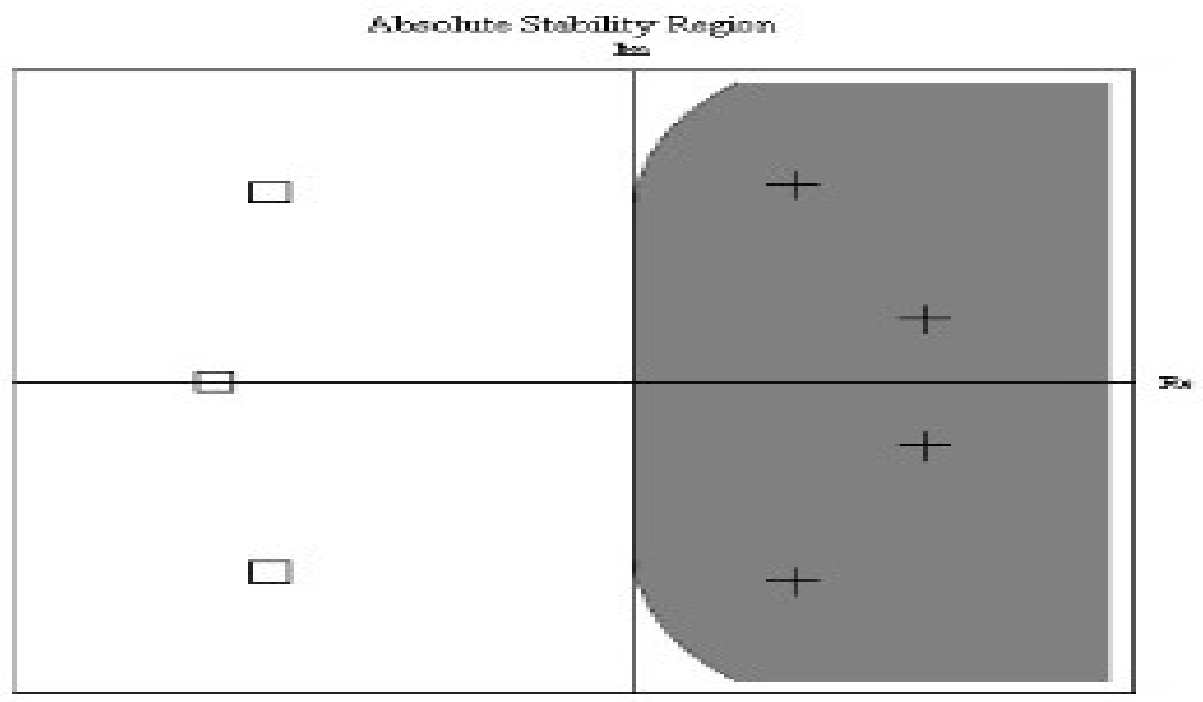

Figure 1. Stability Region of Block Hybrid Multistep Method

\section{Numerical Results}

The following problems are considered to examine the accuracy and computational efficiency of BHMM. The computations were carried out using MATHEMATICA 9.0 software. The following acronyms are used:

- $\mathrm{ES}=$ Exact Solution

- $\mathrm{ESDMM}=$ Error in SDMM

- EMHIRK = Error in MHIRK

- EBHMM $=$ Error in BHMM

- $\mathrm{EESDMM}=$ Error in ESDMM

Problem 1: Consider the non-linear IVP

$$
\begin{gathered}
y_{1}^{\prime}=\lambda y_{1}+y_{2}^{2} \\
y_{2}^{\prime}=-y_{2}
\end{gathered}
$$

with initial conditions

$$
y_{1}(0)=\frac{-1}{\lambda+2}, \quad y_{2}(0)=1,
$$

where $\lambda=10^{4}$ and the exact solution of the IVP is given as

$$
y_{1}(x)=-\frac{e^{-2 x}}{\lambda+2}, \quad y_{2}(x)=e^{-x}
$$

In Table 1, the numerical results obtained using BHMM with $h=10^{-1}$ compares favourably with MHIRK method [10] with $h=10^{-1}$ and is superior to that of Hojjati et al. [20] that used $h=10^{-4}$.
Problem 2: Consider the following stiff problem arising from Chemical Kinetic reactions in a Chemistry experiment.

$$
\begin{gathered}
y_{1}^{\prime}=-0.013 y_{1}-1000 y_{1} y_{2}-2500 y_{1} y_{3} \\
y_{2}^{\prime}=-0.013 y_{1}-1000 y_{1} y_{2} \\
y_{3}^{\prime}=-2500 y_{1} y_{3}
\end{gathered}
$$

with initial conditions

$$
y_{1}(0)=0, \quad y_{2}(0)=1, \quad y_{3}(0)=1
$$

The computed result at $x=2.0$ from the new method BHMM is compared with those of Ismail and Ibrahim (ESDMM [21]), Hojjati et al. SDMM [20], Akinfenwa et al. MHIRK [10]. The step length $h=0.0125$ was used for MHIRK method and the new method BHMM and compared with Ismail and Ibrahim ESDMM [21], Hojjati et al. SDMM [20] with step length $h=0.001$. The result obtained with the new method BHMM is superior to others. See Table 2 for the numerical results.

Problem 3: Consider the non-linear system

$$
\begin{gathered}
y_{1}^{\prime}=-2 y_{1}+y_{2}+2 \sin x \\
y_{2}^{\prime}=998 y_{1}-999 y_{2}+999(\cos x-\sin x)
\end{gathered}
$$

with initial conditions

$$
y_{1}(0)=2, \quad y_{2}(0)=3, \quad 0 \leq x \leq 10
$$

with analytical solution given as

$$
y_{1}(x)=2 e^{-x}+\sin x, \quad y_{2}(x)=2 e^{-x}+\cos x
$$


Table 1. Comparison of Methods for Problem 1

\begin{tabular}{rrrrrr}
\hline$x$ & $y_{i}$ & ES & ESDMM[20] & EMHIRK[10] & EBHMM \\
\hline 3 & $y_{1}$ & $-0.2478257 E-06$ & $2.478147 E-11$ & $3.06450 E-15$ & $5.00564 E-16$ \\
& $y_{2}$ & $0.4978707 E-01$ & $2.471093 E-06$ & $3.07825 E-10$ & $5.02813 E-11$ \\
\hline 5 & $y_{1}$ & $-0.4539085 E-08$ & $3.450217 E-14$ & $9.35475 E-17$ & $1.52787 E-17$ \\
& $y_{2}$ & $0.6737946 E-02$ & $2.304573 E-08$ & $6.94326 E-11$ & $1.13414 E-11$ \\
\hline 10 & $y_{1}$ & $0.3059023 E-12$ & $3.456372 E-18$ & $8.49412 E-21$ & $3.75372 E-20$ \\
& $y_{2}$ & $0.3059023 E-04$ & $3.150734 E-10$ & $9.35666 E-13$ & $1.52836 E-13$ \\
\hline
\end{tabular}

Table 2. Comparison of Methods for Problem 2

\begin{tabular}{rrrrrrr}
\hline$x$ & $y_{i}$ & ES & EESDMM[21] & ESDMM[20] & EMHIRK[10] & EBHMM \\
\hline 2.0 & $y_{1}$ & $-0.361693316929 E-5$ & $0.82 E-10$ & $0.52 E-13$ & $0.110 E-13$ & $2.919 E-15$ \\
& $y_{2}$ & 0.9815029948230 & $0.61 E-05$ & $0.19 E-08$ & $0.220 E-08$ & $5.586 E-10$ \\
& $y_{3}$ & 1.018493388244 & $0.57 E-05$ & $0.63 E-08$ & $0.220 E-08$ & $5.584 E-10$ \\
\hline
\end{tabular}

with exact solution given as

$$
y_{1}(x)=2 e^{-x}-e^{-50 x}, \quad y_{2}(x)=2 e^{-x}+6-e^{-50 x}
$$

The numerical results of Problem 3 are shown in Table 3 using step size $h=10^{-3}$. The derived method integrated the problem efficiently that the numerical solution is close to that of the analytical solution.

\begin{tabular}{rrrr}
\multicolumn{5}{c}{ Table 3. The absolute error for Problem 3 } \\
\hline$x$ & $y_{i}$ & ES & EBHMM \\
\hline 0.25 & $y_{1}$ & 1.805005525 & $4.50751 E-14$ \\
& $y_{2}$ & 2.526513988 & $4.84057 E-14$ \\
0.5 & $y_{1}$ & 1.692486858 & $9.85878 E-14$ \\
& $y_{2}$ & 2.090643881 & $9.81437 E-14$ \\
1.0 & $y_{1}$ & 1.577229867 & $9.45910 E-14$ \\
& $y_{2}$ & 1.276061188 & $9.54792 E-14$ \\
2.0 & $y_{1}$ & 1.179967993 & $1.68310 E-13$ \\
& $y_{2}$ & -0.145476270 & $1.68365 E-13$ \\
4.0 & $y_{1}$ & -0.720171217 & $2.21378 E-13$ \\
& $y_{2}$ & -0.617012343 & $2.23044 E-13$ \\
6.0 & $y_{1}$ & -0.274457993 & $1.01363 E-13$ \\
& $y_{2}$ & 0.9651277911 & $1.01474 E-13$ \\
8.0 & $y_{1}$ & 0.9900291719 & $1.93401 E-13$ \\
& $y_{2}$ & -0.1448291085 & $1.94650 E-13$ \\
10.0 & $y_{1}$ & -0.5439303110 & $6.10623 E-13$ \\
& $y_{2}$ & -0.8389807292 & $6.09068 E-13$ \\
\hline
\end{tabular}

Problem 4: A stiff system of Initial Value Problems

$$
\begin{aligned}
& y_{1}^{\prime}=-8 y_{1}+7 y_{2} \\
& y_{2}^{\prime}=42 y_{1}-43 y_{2}
\end{aligned}
$$

with initial conditions

$$
y_{1}(0)=1, \quad y_{2}(0)=8, \quad x \in[0,15]
$$

Problem 4 was integrated using the step size of $h=10^{-4}$ to aid in comparing with other methods in literature as shown in Tables 4 and 5. It is discovered that BHMM has better accuracy than the others compared with.

Table 4. Numerical Results for Problem 4

\begin{tabular}{rrrr}
\hline$x$ & $y_{i}$ & ES & EBHMM \\
\hline 3 & $y_{1}$ & $9.9574136 \times 10^{-2}$ & $2.68577 \times 10^{-13}$ \\
& $y_{2}$ & $9.9574136 \times 10^{-2}$ & $2.65843 \times 10^{-13}$ \\
6 & $y_{1}$ & $4.9575044 \times 10^{-3}$ & $1.68580 \times 10^{-14}$ \\
& $y_{2}$ & $4.9575044 \times 10^{-3}$ & $1.80611 \times 10^{-14}$ \\
9 & $y_{1}$ & $2.4681961 \times 10^{-4}$ & $7.57646 \times 10^{-15}$ \\
& $y_{2}$ & $2.4681961 \times 10^{-4}$ & $5.43191 \times 10^{-15}$ \\
12 & $y_{1}$ & $1.2288424 \times 10^{-5}$ & $2.10193 \times 10^{-15}$ \\
& $y_{2}$ & $1.2288424 \times 10^{-5}$ & $2.54783 \times 10^{-15}$ \\
15 & $y_{1}$ & $6.1180464 \times 10^{-7}$ & $2.29273 \times 10^{-14}$ \\
& $y_{2}$ & $6.1180464 \times 10^{-7}$ & $1.87085 \times 10^{-14}$ \\
\hline
\end{tabular}

\section{Conclusion}

A one-step L-stable Block Hybrid Multistep Method (BHMM) of order five was developed via interpolation and collocation techniques. The BHMM has the advantage of being self-starting and its L-stable in nature as displayed in figure 1. The block method possesses high accuracy as shown in Tables (1) - (5) where it was compared with some existing methods. The method satisfies the zero-stability, consistency and convergence conditions. BHMM has proved efficient for solving first-order Initial Value Problems. 
Table 5. A Comparison of absolute errors of methods for Problem 4

\begin{tabular}{rrr}
\hline$x$ & Methods & Error $\left|y\left(t_{n}\right)-y_{n}\right|$ \\
\hline 5 & BHMM & $3.1999 \times 10^{-14}$ \\
& TDBDF [22] & $1.5472 \times 10^{-2}$ \\
& TDMM [23] & $1.5476 \times 10^{-2}$ \\
\hline 10 & BHMM & $4.642 \times 10^{-15}$ \\
& TDBDF [22] & $9.0808 \times 10^{-5}$ \\
& TDMM [23] & $9.0808 \times 10^{-5}$ \\
\hline 15 & BHMM & $1.8709 \times 10^{-14}$ \\
& TDBDF [22] & $6.1186 \times 10^{-7}$ \\
& TDMM [23] & $6.1186 \times 10^{-7}$ \\
\hline
\end{tabular}

\section{Acknowledgments}

The authors wish to thank the referees and editor for the comments and suggestions to make this paper a success.

\section{References}

[1] G. G. Dahlquist, "A special stability problem for linear multistep methods", BIT 3 (1963) 27.

[2] J. C. Butcher, The numerical methods for ordinary differential equations, John Wiley and Sons Ltd, Chichester, 2008.

[3] J. D. Lambert, Computational methods for ordinary differential systems: The Initial Value Problems, Wiley, Chichester, 1973.

[4] W. H. Enright, "Second derivative multistep methods for stiff ODEs", SIAM Journal of Numerical Analysis 11 (1974) 321.

[5] B. I. Akinnukawe \& S. A. Okunuga, "A Seventh-order block integrator for solving stiff systems", Nigerian Journal of Mathematics and Applications 24 (2015) 67.

[6] C. W. Gear, "Hybrid methods for initial value problems in ordinary differential equations", SIAM Journal of Numerical Analysis 2 (1965) 69.

[7] S. A. Okunuga, "A fourth order composite two step method for stiff problems", International Journal of Computer Mathematics 72 (1999) 39.

[8] J. R. Cash, "On the integration of stiff systems of ODEs using Extended Backward Differentiation Formulae", Numerische Mathematik 34 (1980) 235.
[9] O. A. Akinfenwa, B. Akinnukawe \& S. B. Mudasiru, "A family continuous third derivative block methods for solving stiff systems of first order ODEs", Journal of the Nigerian Mathematical Society 34 (2015) 160.

[10] O. A. Akinfenwa, S. A. Okunuga, B. I. Akinnukawe, U. P. Rufai \& R. I. Abdulganiy, "Multi-derivative hybrid implicit Runge-Kutta method for solving stiff system of a first order differential equation", Far East Journal of Mathematical Sciences (FJMS) 106 (2018) 543.

[11] A. O. Adesanya, R. O. Onsachi \& M. R. Odekunle, "New algorithm for first order stiff initial value problem", Fasciculi Mathematici 58 (2017) 2.

[12] A. P. Ijezie \& K. O. Muka, "Modified SDBDF based on a non-zero root of the second characteristics polynomial", Journal of Science and Technology Research 1 (2019) 128.

[13] J. D. Lambert, Numerical methods for ordinary differential systems, John Wiley, New York, 1991.

[14] P. Onumanyi, U. W. Sirisena \& S. N. Jator. Continuous finite difference approximations for solving differential equations, "International Journal of Computational Mathematics", 72 (1999) 15.

[15] W. E. Milne, Numerical solution of differential equations, John Wiley, New York, 1953.

[16] D. Sarafyan, Multistep methods for the numerical solution of ODEs made self-starting, Technical Report No. 495, Mathematics Research Center, Madison, Wisconsin, 1965.

[17] L. F. Shampine \& H. A. Watts, "Block implicit one-step methods", Mathematics of Computation 23 (1969) 731.

[18] S. O. Fatunla, "Block methods for second order IVPs", Internal Journal of Computational Mathematics 41 (1991) 55.

[19] P. Henrici, Discrete Variable Methods in ODEs, John Wiley, New York, 1962.

[20] G. Hojjati, M. Y. Rahimi-Ardabili \& S. M. Hosseini, "New second derivative multistep methods for stiff systems", Applied Mathematical Modelling 30 (2006) 466.

[21] G. A. Ismail \& I. H. Ibrahim, "New efficient second derivative multistep methods for stiff systems", Applied Mathematical Modelling 23 (1978) 279.

[22] R. I. Okuonghae, M. N. O. Ikhile \& J. Osemeke, "An off-step-point methods in multistep integration of stiff ODEs", NMC Journal of Mathematical Sciences 3 (2014) 731.

[23] A. K. Ezzeddine \& G. Hojjati, "Third derivative multistep methods for stiff systems", International Journal of Nonlinear Science 14 (2012) 443. 